\title{
Spontaneous Retropharyngeal Space Hematoma: How to Manage?
}

\author{
Y. Lakhdar, D. Berrada Elazizi, M. Elbouderkaoui, Y. Rochdi, and A. Raji
}

\section{ABSTRACT}

Introduction: Spontaneous Retropharyngeal Hematoma (SRH) is a rare affection which occurs without traumatism.

Case report: A 68 years old woman brought to the emergency department with a cervico-thoracic swelling rapidly increasing in size without any previous traumatic history or anticoagulant medication. Complicated by dysphagia and dysphonia without signs of dyspnea. The clinical examination revealed a painful anterior cervico-thoracic swelling with an ecchymotic cupboard on it. Cervico-thoracic CT scan found a voluminous retropharyngeal collection, spontaneously hyperdense, extended to the oropharynx and antero-superior mediastinum without further lesions. Biological assessment was without abnormalities. The diagnosis of retropharyngeal hematoma was retained. The treatment was based on corticosteroids and antibiotics drugs with strict clinical and radiological monitoring. With conservative treatment, the evolution was favorable, marked by progressive regression of the hematoma until its disappearance and an absence of recurrence after a retreat of one year.

Conclusion: The clinical presentation of a retropharyngeal hematoma out of an evocative context is misleading and lead to radiological investigation without delaying the treatment which varies from supervision to surgery according to respiratory status and evolution under strict control.

Keywords: hematoma, retropharyngeal space, dyspnea.

\section{INTRODUCTION}

Spontaneous Retropharyngeal Haematoma (SRH) is a rare affection which occurs without traumatism and difficult to diagnose early. It is a diagnostic and therapeutic emergency that can be life-threatening.

\section{OBJectives}

We report a rare case of an elderly woman who presented a spontaneous retropharyngeal hematoma, and we discuss ethiopathogeny, clinic's and therapeutic's management.

\section{CASE REPORT}

Mrs. A. F. 68 years old, diabetic, brought to the emergency department for a cervico-thoracic swelling evolving for two days rapidly increasing in size with appearance of bruise extended upon it. The patient reports compressive signs of dysphagia and dysphonia without dyspnea. There was no specific past medical history such as a trauma, upper respiratory infection, administration of anticoagulants or other drugs, or any other medical condition.

The clinical examination found a patient who was conscious, hemodynamically and respiratory stable with a painful anterior cervico-thoracic swelling with an ecchymotic
Submitted : March 5, 2021

Published : March 27, 2021

ISSN: $2593-8339$

DOI: $10.24018 /$ ejmed.2021.3.2.755

\section{Y. Lakhdar*}

ENT department, Mohammed VI University Hospital Center, Marrakech, Morocco.

(e-mail: lakhdar.orl@gmail.com) D. Berrada Elazizi

ENT department, Mohammed VI University Hospital Center, Marrakech, Morocco.

(e-mail: douniaberradaelazizi@ gmail.com) M. Elbouderkaoui

ENT department, Mohammed VI University Hospital Center, Marrakech, Morocco.

(e-mail: med.elbouderkaoui@ gmail.com ) Y. Rochdi

ENT department, Mohammed VI University Hospital Center, Marrakech, Morocco.

A Raji

ENT department, Mohammed VI University Hospital Center, Marrakech, Morocco.

*Corresponding Author

cupboard on it (Fig. 1). The oropharynx was unremarkable without bulging or visible mass.

The nasofibroscopy of upper airways showed a slight ecchymotic oedema of the laryngopharynx with a good laryngeal stream and a minimal salivary stasis.

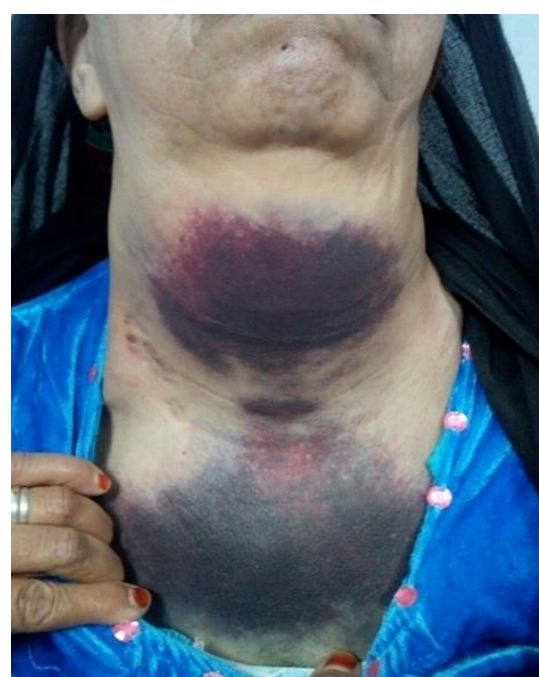

Fig. 1. Cervico-thoracique swelling with ecchymotic cupboard.

An urgently needed cervico-thoracic computed tomography scan (CT) revealed a voluminous spontaneously hyperdense retro-pharyngeal collection, extending from the oropharynx to the anterosuperior mediastinum, pushing the 
larynx, trachea, and esophagus forward, without further lesions (Fig. 2 and 3).

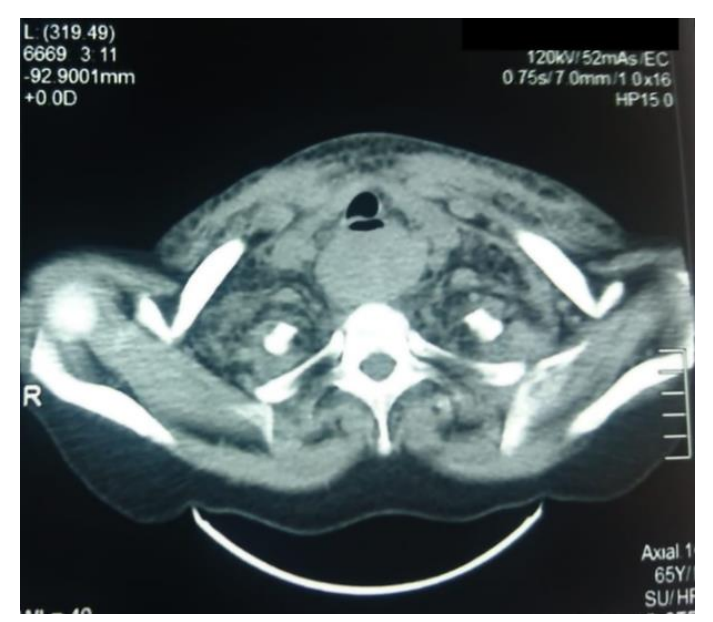

Fig. 2. Axial cervico-thoracic CT scan showing a spontaneously hyperdense lesion of the retropharyngeal space evoking a hematoma.

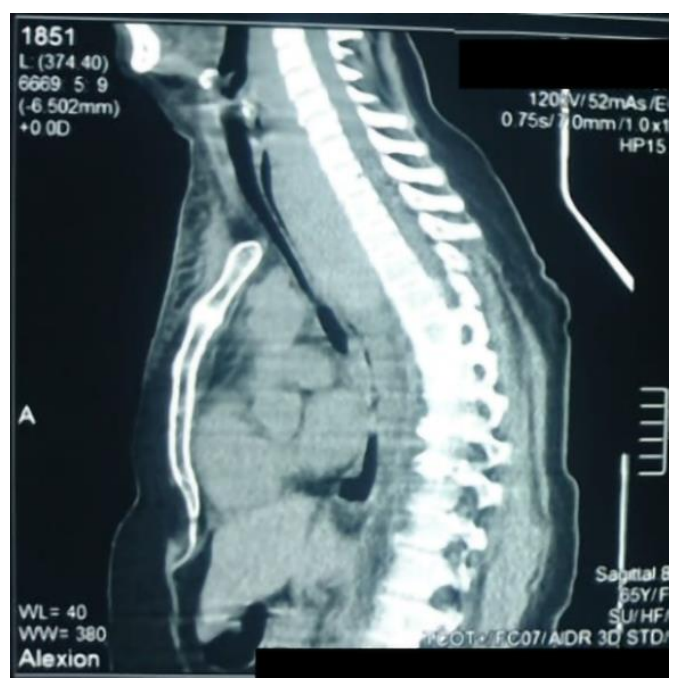

Fig. 3. Sagittal cervico-thoracic CT scan showing a spontaneously hyperdense lesion of the retropharyngeal space evoking a hematoma.

Based on clinical and radiologic findings, a diagnosis of spontaneous retropharyngeal hematoma was made.

Biological samples were as follow: Prothrombin level = $96 \%$, activated partial thromboplastin time $=30 \%$, platelets count $=180000 / \mathrm{mm}^{3}$, hemoglobin level=12.9 g/dl, white blood cell count $=6572 / \mathrm{mm}^{3}$, bleeding test $=3$, , normal aggregation platelet test and no deficit of coagulation factors.

Magnetic resonance imaging with angiographic sequences did not detect any abnormality especially in the thyroid and cervico-facial vessels.

In front of the absence of threatening respiratory signs, we opted for conservative treatment with intraveinous corticosteroids and antibiotics. The patient was closely followed up with strict clinical and biological monitoring.

A CT scan control, redone on the seventh day of hospitalization, showed a decrease in the size of the retpharyngeal collection. Clinical evolution was favorable, marked by the stability of the hemodynamic constants and by the progressive regression of the cervical hematoma until its total disappearance after two months. Regular monitoring and control did not report recurrence even after one year of follow up.

\section{DISCUSSION}

The retro-pharyngeal space hematoma is rare. It is particularly dangerous by its location, which can compress the upper airways and threaten the vital prognosis.

This diagnosis remains difficult outside of an evocative context. A classic "Capp's triad" is described associating oesophago-tracheal compression, anterior displacement of the trachea, and anterior cervicothoracic bruise. The patient usually consults for odynophagia or cervical pain [1]-[3]. The examination finds a pharyngeal bulge and ecchymosis of the tracheal and pharyngeal mucosa. However, the reasons for consultation and the clinic for HRP vary. Dyspnea, the most alarming sign, is sometimes absent especially at the beginning [4]. In the same way the cervical bruise is not a constant sign [4].

The diagnosis of hematoma can be made as early as a few hours after the acute event, when hyperintensity is seen on both T1- and T2-weighted MRI sequences, and this diagnosis is reinforced when changes due to magnetic susceptibility effects are demonstrated on gradient-echo sequences [2]. Differential diagnosis of retropharyngeal hematoma includes essentially a pre vertebral abscess complicating tuberculous spondylodiscitis [1], [2], [4].

The etiologies of HRP are numerous. The most common are cervical trauma, coagulopathies, invasive explorations and foreign body ingestion [5], [6]. Infectious causes have been incriminated [7]. In very rare cases, HRP is due to bleeding from a cervical organ such as the thyroid [5], [8]. Other possible etiologies are intrathoracic overpressure during cough [9], or Valsalva maneuver [5].

The therapeutic management of HRP is poorly coded. In case that the hematoma is very extensive or exuberant causing sign of respiratory distress, it is imperative to perform intubation or tracheotomy. However, if there is a clear situation, the indication of a gesture on upper airways is controversial. Some authors advocate prophylactic intubation or tracheostomy because evolution remains unpredictable [9]. Others report that it is necessary only in case of aggravation because on the one hand the hematoma usually resolves in one to two weeks and on the other hand, they can exacerbate the bleeding [9]. This attitude is only recommended if strict supervision is available. Surgical evacuation is mainly indicated if the obstruction is important and life-threatening or if the hematoma is rapidly expansive [10]. Surgical exploration is necessary in case of confirmed vascular lesions [4]. The use of steroids and antibiotics is common although their interest has not been demonstrated.

\section{CONCLUSION}

The clinical presentation of a retropharyngeal hematoma out of an evocative context is misleading but high clinical suspicion is warranted in patients who present with acute dysphagia, odynophagia, and dysphonia and lead to radiological investigation without delaying the treatment which varies from supervision to surgery depending on the hemodynamic and respiratory status at admission and depending on the clinical course. 
European Journal of Medical and Health Sciences www.ejmed.org

\section{CONFLICT OF INTEREST}

All authors declared that there is no conflict of interests.

\section{CONSENT}

Informed consent was obtained from the patient for publication of this case report and accompanying images.

\section{REFERENCES}

[1] E C. Fox, V Manchala. Retropharyngeal Hematoma as an Unusual Presentation of Myelodysplastic Syndrome: A Case Report. Am J Case Rep. 2018; 19: 969-972.

[2] Munoz A, Fischbein NJ, De Vergas J. Spontaneous retropharyngeal hematoma: Diagnosis by MR Imaging AJNR. 2001;22:1209-11.

[3] Cohen JI. Spontaneous cervical hemorrhage with near-complete airway obstruction. Head and Neck 1998;20: 350-53

[4] Bapat VN, Brown K, Nakas A. Retropharyngeal hematoma-a rare complication of anticoagulant therapy. Eur.J.Cardio-thoracic Surg.2002;21:117-18.

[5] Vinidh P, Maroju R.S, Ali M.S Spontaneous retro and parapharyngeal haematoma caused by intrathyroid bleed. J. Laryngol. Otol. 2002;116: 854-58.

[6] Tomohiro Yamamoto, MD, PhD; Martin Schmidt-Niemann, Dr. med; Ehrenfried Schindler, Dr. med. A Case of Acute Upper Airway Obstruction in a Pediatric Hemophilia A Patient Because of Spontaneous Retropharyngeal Hemorrhage, annemergmed, Volume 67, Issue 5, pp. 616-619.

[7] Jones TM, Owen GO, Morar P.Spontaneous retropharyngeal haematoma attributable to Ebstein-Barr virus infection.J Laryngol Otol. 1996;110:1075-77.

[8] Armstrong WB, Funk GF, Rice DH. Acute airway compromise secondary to traumatic thyroid hemorrhage Arch Otolaryngol Head Neck Surgery 1994;120:427 30.

[9] Bloom DC, Haegen T, Keefe MA Anticoagulation and spontaneous retropharyngeal hematoma .J. Em Med. 2003;24:389-94.

[10] Senthuran S, Lim S, Gunning KE/ Life-threatening airway obstruction caused by a retropharyngeal haematoma.Anaesthesia 1999;54:674. 